\title{
Clinical application of expectorant therapy in chronic inflammatory airway diseases (Review)
}

\author{
TING ZHANG and XIANGDONG ZHOU \\ Department of Respiratory Medicine, The Second Affiliated Hospital, \\ Chongqing Medical University, Chongqing 400010, P.R. China
}

Received August 31, 2013; Accepted January 14, 2014

DOI: $10.3892 /$ etm.2014.1494

\begin{abstract}
Airway mucus hypersecretion is a significant clinical and pathological feature of chronic inflammatory airway diseases. Its clinical presentations include recurrent coughing and phlegm. Airway mucus is closely associated with the occurrence, development and prognosis of chronic inflammatory airway diseases and critically affects the lung function, quality of life, hospitalization rate and mortality of patients with chronic inflammatory airway diseases. Therefore, expectorant therapies targeting the potential mechanisms of mucus hypersecretion have been the focus of numerous studies. Conventional expectorants are mainly mucoactive medicines, including nausea-stimulating expectorants, mucolytics, mucokinetics, and proteases and nucleases. In addition, certain traditional Chinese herbal medicines and non-mucoactive agents, including muscarinic acetylcholine receptor antagonists, corticosteroids, leukotriene receptor antagonists and macrolide antibiotics, have also shown expectorant effects. Several novel medicines for expectorant therapy have emerged, including cholesterol-lowering statins, epidermal growth factor receptor tyrosine kinase inhibitors, phosphodiesterase-4 inhibitors, stanozolol, surfactants, flavonoids, tachykinin receptor antagonists, protease inhibitors, cytokine antagonists and purinergic agonists. With the increasing number of multidisciplinary studies, the effectiveness of expectorant therapy for the treatment of chronic inflammatory airway diseases has been confirmed. Therefore, the development of novel expectorants and the standardization of expectorant therapy are the direction and focus of future studies, thus benefiting patients who have a chronic inflammatory airway disease.
\end{abstract}

Correspondence to: Professor Xiangdong Zhou, Department of Respiratory Medicine, The Second Affiliated Hospital, Chongqing Medical University, 74 Linjiang Road, Yuzhong, Chongqing 400010, P.R. China

E-mail: zxd999@263.net

Key words: airway mucus hypersecretion, chronic inflammatory airway disease, expectorant therapy

\section{Contents}

1. Introduction

2. Pathological changes of airway mucus hypersecretion and the association with respiratory diseases

3. Classification and mechanisms of common expectorants

4. Chinese herbal expectorants

5. Novel types of expectorants

6. Summary

\section{Introduction}

Airway mucus hypersecretion is one of the important clinical and pathological features of chronic inflammatory airway diseases, including chronic obstructive pulmonary disease (COPD), bronchial asthma, bronchiectasis and cystic fibrosis (1-3). Long-term and repeated inflammatory stimuli of chronic airway inflammation induce the metaplasia of goblet cells, as well as the hyperplasia and hypertrophy of submucosal glands, causing a 'secretory hyperresponsiveness' that leads to impaired mucociliary clearance. Consequently, excessive mucus is more difficult to expel and is retained in the airways to form mucous plugs, which worsen the obstruction of the already narrowed airway and increase the colonization by pathogenic bacteria. These events may lead to persistent inflammation, sustained hypoxia, deterioration of the condition of a patient or even mortality (4-6). It has become increasingly clear that airway mucus hypersecretion is not only a clinical symptom but also an independent risk factor for disease progression and the poor prognosis of patients with chronic inflammatory airway diseases (7-9). Therefore, chronic inflammatory airway diseases with airway mucus hypersecretion has become a central issue in a number of studies (10-13). In the present study, the significance of expectorant therapy and the classification of common and novel expectorants are briefly reviewed.

2. Pathological changes of airway mucus hypersecretion and the association with respiratory diseases

Airway mucus is a heterogeneous mixture of glycoproteins, non-glycoprotein-like proteins, lipids, minerals, water and other compounds. Although the components and corresponding proportions in airway mucus vary greatly under different 
conditions, mucin, a highly glycosylated macromolecule, is always the major component and determines the hydrophilic and viscoelastic properties of airway mucus. The apparently elevated glycosylation and sulfation of mucus during chronic airway inflammation significantly increases the viscosity and acidity of airway mucus, making it immobile in the airway and thus more difficult to expel, which further aggravates the airway obstruction (14-16). A long-term study conducted by the European Community Respiratory Health Survey II revealed that in individuals with secretory hyperresponsiveness symptoms, including chronic cough and phlegm, the incidence of COPD is 2.88-fold higher than that in the general population and is not associated with smoking exposure (17). Burgel and Martin also confirmed that the incidence of acute exacerbation and readmission was markedly increased in patients with COPD with a cough and expectoration (9). Vestbo and Hogg identified that anti-inflammatory treatment alone was insufficient for the treatment of chronic inflammatory airway diseases, whereas expectorant therapy was not only able to significantly alleviate the clinical symptoms but also helped to reduce the respiratory inflammatory response (18). Delmotte et al determined that inflammatory cytokines, such as tumor necrosis factor- $\alpha$, significantly enhanced the enzymatic activity of sulfotransferases and glycosyltransferases, which is consistent with the observation of increased glycosylation of mucin in patients with an inflammatory airway disease (19). A follow-up study, based on 101 patients with COPD (GOLD stage III-IV) who underwent lung volume reduction surgery, revealed that the severity of the airflow limitation induced by mucus hypersecretion was closely associated with the mortality of patients (20). Therefore, airway mucus hypersecretion plays an important role in the occurrence and development of chronic inflammatory airway diseases, and has been identified to be associated with the lung function, quality of life, hospitalization rate and mortality of patients with chronic inflammatory airway diseases. Consequently, expectorant therapy that is based on the potential mechanism of mucus hypersecretion has become an important target for the treatment of chronic inflammatory airway diseases.

\section{Classification and mechanisms of common expectorants}

Clinically available expectorant medicines are aimed at inhibiting the production and secretion of mucins, reducing the viscoelasticity of mucus, rehabilitating the normal structure and function of the mucus layer, improving mucociliary clearance and accelerating the transport of mucus. Accordingly, they are known as mucoactive agents $(21,22)$, and are further divided into: i) Nausea-stimulating expectorants, such as guaifenesin; ii) mucolytics, such as ambroxol, which cleave mucopolysaccharide fibers, and N-acetylcysteine (NAC) and carbocisteine, which cleave disulfide bonds; iii) mucokinetics, such as myrtle oil, a powerful thinner of hardened mucus; and iv) proteases and nucleases, such as $\alpha$-chymotrypsin. Of these classes, mucolytics and mucokinetics are primarily used, particularly ambroxol which accounts for almost $70 \%$ of the expectorant treatment in China (23).

As the most extensively used expectorant medicine in clinical practice in China, ambroxol has a very wide range of effects on the respiratory system. It is able to reduce the viscosity of sputum by inducing the bronchial glands to secrete serum and breaking up the mucopolysaccharide fibers of the mucin, which facilitates the penetration of antibiotics into the mucus and improves the local antibacterial effect. Simultaneously, ambroxol also induces alveolar type II cells to synthesize and secrete pulmonary surfactants that reduce the adhesion of the mucus to the cilia and accelerate the transport of mucus in the airway, which helps to expel the sputum and increase the airway mucosal clearance. In addition, ambroxol has specific antitussive, antioxidant and anti-inflammatory effects, along with a relatively significant inhibitory effect on histamine-induced constriction of the bronchial smooth muscle. Furthermore, it may also be used to prevent hyaline membrane disease in premature infants and to alleviate nitrosourea-induced pulmonary toxicity during the chemotherapy of malignant brain tumors $(24,25)$.

As a mucolytic agent, the clinical efficacy of NAC has gained recognition and attention. It reduces the viscosity of mucus by cleaving the disulfide bonds of mucins and the DNA fibers in the purulent sputum, leading to its efficacy under conditions where general expectorant medicines are ineffective. It also accelerates the ciliary movement within airway mucosa and stimulates the gastro-pulmonary vagal reflex, thereby promoting the excretion of mucus. In addition, NAC has comprehensive antioxidant, anti-inflammatory, anti-injury, anti-lipid oxidation, anti-platelet aggregation, anti-mutagenesis and vasodilatory activities, and is able to protect anti-protease activity and inhibit allergic reactions, among others. (26-28). Pela et al demonstrated that the prolonged treatment of patients with COPD with NAC (400 mg/day for six months) led to a significant improvement in the forced expiratory volume in $1 \mathrm{sec}$ and maximum expiratory flow at 50\% of the forced vital capacity, accompanied by a significant reduction in exacerbations of $41 \%$ (29). Similarly, Gerrits et al concluded that a daily intake of $400 \mathrm{mg}$ NAC has a significant protective effect and reduces the readmission rate of patients with COPD by $30 \%$ (30). In addition to being a safe and efficacious expectorant medicine, NAC has been extensively used to treat pulmonary diseases including emphysema, tuberculosis, fibrous alveolitis and primary pulmonary amyloidosis, as well as diseases of the cardiovascular and central nervous system and acquired immune deficiency syndrome (31).

Carbocisteine, as a mucoregulatory agent, recovers the viscoelasticity of normal mucus and enhances mucociliary clearance via interactions between the disulfide bonds of mucin and the carboxymethyl structure of the agent. The antioxidant and anti-inflammatory protective effects of carbocisteine on bronchial cells are also relevant to its efficacy in the treatment of chronic airway inflammatory diseases (32). The PEACE study on the efficacy of carbocisteine has shown that, although carbocisteine did not significantly improve the lung function and blood oxygen saturation of patients with COPD, the medicine significantly reduced the frequency of acute exacerbations of COPD (AECOPD) and improved health-related quality-of-life scores compared with those of the control group. Therefore, the authors recommended that carbocisteine is used for the prevention and treatment of AECOPD (33). Other studies have also indicated that carbocisteine is able to improve the quality of life of patients with COPD $(34,35)$. 
Although the longstanding and popular therapeutic option for the treatment of chronic inflammatory airway diseases is mucoactive medicines, numerous non-expectorant agents have also received great attention as a type of mucus therapy. The agents that are commonly available for clinical use include muscarinic receptor blockers, glucocorticosteroids, leukotriene receptor antagonists, macrolide antibiotics, $2-3 \%$ hypertonic sodium chloride and $2-7 \%$ hypertonic sodium bicarbonate solution. Each of them inhibits the excessive production and/or secretion of airway mucins via different mechanisms despite their common treatment functions $(22,36)$.

\section{Chinese herbal expectorants}

The role of herbal medicine is significant in expectorant management. Balloon flower, rhizoma arisaematis, Caladium, Polygala and Aster are commonly adopted for expectorant therapy. Triterpenoid saponins are considered as the major effective ingredients. These Chinese herbal medicines are mainly nausea-stimulating expectorants, which induce mild nausea by stimulating the gastric mucosa and stimulate the respiratory gland cells to increase the secretion of thinner sputum, thus facilitating its expulsion. Chinese herbal expectorants have a confirmed therapeutic effect, and their clinical application is promising. However, a number of issues remain to be improved, such as variations to prescriptions and over-dispersive studies. Therefore, further exploration of highly efficacious and rapid-onset agents is required for the development of novel medications for oral administration (37-39).

\section{Novel types of expectorants}

Statin-like cholesterol-lowering medicines. Statins, such as simvastatin, are a class of medicines used to lower cholesterol levels. A number of studies have identified that they are also able to modulate airway inflammatory processes by inhibiting the expression of a variety of inflammatory factors and inducing the apoptosis of inflammatory cells, which helps to inhibit inflammation-induced airway mucus hypersecretion. Further, the anti-inflammatory effect of statins has been verified to be independent of their lipid-lowering role. A few studies have suggested that the anti-inflammatory effect of statins is directly associated with their function of promoting the apoptosis and clearance of inflammatory cells (40-42). It has also been demonstrated that statins are able to reduce the morbidity and mortality of patients with COPD $(43,44)$, suggesting that the combined effects of statins on COPD may lead to them becoming a novel therapy option for chronic airway inflammatory diseases.

Epidermal growth factor receptor (EGFR) tyrosine kinase inhibitors. The EGRF signaling pathway is a common pathway for numerous inflammatory mediators leading to mucus overproduction in human airways. Therefore, blocking this pathway provides a novel treatment target for airway mucus hypersecretion and reduces sputum retention. Clinical case studies have identified that the selective EGFR tyrosine kinase inhibitor gefitinib (Iressa), which is used to treat non-small-cell lung cancer, markedly inhibited airway mucus hypersecretion in patients with lung cancer. Further, the suppressive effect on airway mucus hypersecretion occurred prior to and more markedly than the inhibition of the growth of the tumor (45-47).

Phosphodiesterase-4 inhibitors. As a major metabolic enzyme of cyclic adenosine monophosphate during the inflammatory response, phosphodiesterase-4 plays an important regulatory role in the synthesis and release of airway non-adrenergic non-cholinergic neurotransmitters. Suppression of phosphodiesterase-4 reduces the release of inflammatory mediators and downregulates inflammatory responses and thereby the airway mucus hypersecretion. The novel selective phosphodiesterase-4 inhibitors cilomilast and roflumilast inhibit the activation of numerous inflammatory and immune cells in chronic inflammatory diseases. Therefore, their anti-inflammatory effect indicates positive prospects for phosphodiesterase-4 inhibitors in the management of airway mucus hypersecretion (48-50)

Stanozolol-related medicines. Stanozolol-related medicines, including erdosteine, fudosteine and lifusteine, play important roles in regulating the composition, properties and motion of mucus. These medicines are able to promote mucociliary transport, reduce the stimulation of mucus secretion, protect $\alpha 1$-antitrypsin protease against the activation of protease, specifically human neutrophil elastase, and significantly increase the concentration of antimicrobial agents in the sputum (as well as synergistically regulating the local activity of those agents). They are have anti-inflammatory activity, aid mucosal repair, suppress goblet cell hyperplasia and promote serous secretion (51-54). Therefore, this class of medicines is ideal for the comprehensive treatment of chronic inflammatory airway diseases, particularly COPD.

Surfactants. Tyloxapol is representative of this class of medicines and decreases the surface tension of sputum, thereby reducing its viscosity and facilitating expectoration.

Flavonoids. Flavonoids are natural ingredients that are extracted from plants with similar biological activities. A considerable number of studies conducted on animals, as well as in vitro, have demonstrated the diverse beneficial biological activities of flavonoids, including anti-inflammatory, antioxidative and antibacterial activities and the induction of apoptosis. All of these effects are beneficial for the prevention and treatment of pulmonary diseases. Theaflavins, exocarpium citri grandis, Forsythia, breviscapine, naringenin, farrerol and quercetin are typical clinically used flavonoids and flavonoid-containing therapeutic agents (55-57). Due to their low costs and high availability from natural sources, these medicines have great potential for development as medicines for chronic inflammatory airway diseases.

Tachykinin receptor antagonists. Tachykinins, including neuropeptide A, neuropeptide B and substance $\mathrm{P}$, are a class of non-adrenergic non-cholinergic neurotransmitter that can mediate neurogenic mucin secretion via neurokinin 1 receptors. A long-acting tachykinin receptor antagonist, MEN11467, inhibits the secretory response (58), suggesting its therapeutic potential for airway mucus hypersecretion

Protease inhibitors. Neutrophil elastase is the most powerful inflammatory stimulus currently known to cause the significant synthesis and secretion of mucin (59). Studies on airway mucus hypersecretion in asthma have revealed that 
the neutrophil elastase inhibitors ONO-5046 and ICI 200,355 are able to suppress ozone-induced and ovalbumin sensitization-induced goblet cell hypersecretion and neutrophil accumulation, respectively $(60,61)$. The protease inhibitor sivelestat is now available for the clinical treatment of acute pulmonary injury. Furthermore, the future treatment of airway diseases with sivelistat (or Sivelestat), a small molecule inhibitor of neutrophilelastase, is planned (62).

Cytokine antagonists. Various types of cells and cytokines are involved in the complex pathological process of chronic airway inflammation and stimulate the abnormal production and secretion of mucin in bronchial epithelial cells. Bacillus Calmette-Guérin (BCG), as a nonspecific immunomodulator, is a type of powerful immune stimuli. It increases the expression levels of Th1-like cytokines (e.g. interferon- $\gamma$ and interleukin (IL)-12), inhibits the expression of Th2-like cytokines, reduces the accumulation and infiltration of eosinophils in airways, and suppresses the hyperplasia and metaplasia of goblet cells as well as the resultant mucus production and secretion. Therefore, BCG achieves preventive and therapeutic results (63). Furthermore, studies on antagonists of other types of cytokines (e.g. IL-4, IL-9 and transforming growth factor- $\beta$ ) are in progress (64-66).

Purinergic agonists. The purine-sensitive receptor, $\mathrm{P} 2 \mathrm{Y} 2$, has a comprehensive role in the regulation of the steady state of the airway mucus layer. The P2Y2 receptor, when activated, enhances the transmembrane movement of water molecules, thins out mucins and accelerates cilial transport mobility. P2Y2 receptor agonists include purine nucleoside triphosphates, pyrimidine nucleosides and INS365 (67). Studies have indicated that inhaled pyrimidine nucleosides and INS365 are capable of accelerating the mucus flow rate in airways and enhancing mucociliary clearance; another P2Y2 agonist, INS37217, has activity similar to that of pyrimidine nucleosides $(68,69)$.

\section{Summary}

With the continued exploration of the role of airway mucus hypersecretion in the pathogenesis of chronic inflammatory airway diseases, the results confirm from an evidence-based medicine point of view that patients with chronic inflammatory airway diseases benefit from treatment with expectorants (70). The crucial role of timely and effective expectorant intervention for the treatment of chronic airway inflammatory disease has been widely accepted by clinicians. However, controversies remain over the applicable subjects, the reasonable dosage and the duration of treatment, among other therapeutic considerations. The phenomenon of chronic airway mucus hypersecretion (presenting as a cough and expectoration) is ubiquitous in all chronic airway inflammatory diseases. Therefore, a study has suggested that therapies targeting mucus hypersecretion in chronic airway inflammation should be recommended regardless of the presence of a chronic cough and expectoration (9). Thus, future studies on the mechanism of expectorant medicines and the exact role expectorant medicines should play in the standard treatment of chronic airway inflammatory diseases are required. The development of novel expectorant medicines and an optimal therapeutic regimen is required.

\section{Acknowledgements}

This review was supported by grants from the National Natural Science Foundation of China (no. 31171346; 81070031; 81100 003; 81370111; and 31211120168).

\section{References}

1. Lundgren JD and Shelhamer JH: Pathogenesis of airway mucus hypersecretion. J Allergy Clin Immunol 85: 399-417, 1990.

2. Rogers DF: Mucus hypersecretion in chronic obstructive pulmonary disease. Novartis Found Symp 234: 65-83, 2001.

3. Zhou X, Tong J and Lan J: Chronic obstructive pulmonary disease airway mucin molecular phenotypic studies. Chin J Tuberc Respir Dis 25: 437-441, 2002 (In Chinese).

4. Vestbo J: Epidemiological studies in mucus hypersecretion. Novartis Found Symp 248: 3-19, 277-282, 2002.

5. Rogers DF: Physiology of airway mucus secretion and pathophysiology of hypersecretion. Respir Care 52: 1134-1149, 2007.

6. Davis CW and Dickey B: Regulated airway goblet cell mucin secretion. Annu Rev Physiol 70: 487-512, 2008.

7. Rogers DF: Airway mucus hypersecretion in asthma: an undervalued pathology? Curr Opin Pharmacol 4: 241-250, 2004.

8. Cerveri I and Brusasco V: Revisited role for mucus hypersecretion in the pathogenesis of COPD. Eur Respir Rev 19: 109-112, 2010.

9. Burgel PR and Martin C: Mucus hypersecretion in COPD: should we only rely on symptoms? Eur Respir Rev 19: 94-96, 2010.

10. Lai H and Rogers DF: New pharmacotherapy for airway mucus hypersecretion in asthma and COPD: targeting intracellular signaling pathways. J Aerosol Med Pulm Drug Deliv 23: 219-231, 2010.

11. Rogers DF: Mucoactive agents for airway mucus hypersecretory diseases. Respir Care 52: 1176-1197, 2007.

12. Henke MO, Shah SA and Rubin BK: The role of airway secretions in COPD - clinical applications. COPD 2: 377-390, 2005.

13. Disse B: Clinical evaluation of new therapies for treatment of mucus hypersecretion in respiratory diseases. Novartis Found Symp 248: 254-276, 277-282, 2002.

14. Evans CM and Koo JS: Airway mucus: the good, the bad, the sticky. Pharmacol Ther 121: 332-348, 2009.

15. Voynow JA and Rubin BK: Mucins, mucus, and sputum. Chest 135: 505-512, 2009

16. Morcillo EJ and Cortijo J: Mucus and MUC in asthma. Curr Opin Pulm Med 12: 1-6, 2006.

17. de Marco R, Accordini S, Cerveri I, et al: Incidence of chronic obstructive pulmonary disease in a cohort of young adults according to the presence of chronic cough and phlegm. Am J Respir Crit Care Med 175: 32-39, 2007.

18. Vestbo J and Hogg JC: Convergence of the epidemiology and pathology of COPD. Thorax 61: 86-88, 2006.

19. Delmotte P, Degroote S , Lafitte JJ, et al: Tumor necrosis factor alpha increases the expression of glycosyltransferases and sulfotransferases responsible for the biosynthesis of sialylated and/or sulfated Lewis x epitopes in the human bronchial mucosa. J Biol Chem 277: 424-431, 2002.

20. Hogg JC, Chu FS, Tan WC, et al: Survival after lung volume reduction in chronic obstructive pulmonary disease: insights from small airway pathology. Am J Respir Crit Care Med 176: 454-459, 2007.

21. Rogers DF and Barnes PJ: Treatment of airway mucus hypersecretion. Ann Med 38: 116-125, 2006.

22. Barnes PJ: Current and future therapies for airway mucus hypersecretion. Novartis Found Symp 248: 237-253, 277-281, 2002.

23. Lun Z: The market analysis of Ambroxol in China. Chinese Pharmaceutical Information, 22: 23-26, 2006 (In Chinese).

24. Beeh KM, Beier J, Esperester A and Paul LD: Antiinflammatory properties of ambroxol. Eur J Med Res 13: 557-562, 2008.

25. Malerba M and Ragnoli B: Ambroxol in the 21st century: pharmacological and clinical update. Expert Opin Drug Metab Toxicol 4: 1119-1129, 2008.

26. Dekhuijzen PN and van Beurden WJ: The role for $\mathrm{N}$-acetylcysteine in the management of COPD. Int J Chron Obstruct Pulmon Dis 1: 99-106, 2006.

27. Sadowska AM, Manuel-Y-Keenoy B and De Backer WA: Antioxidant and anti-inflammatory efficacy of NAC in the treatment of COPD: discordant in vitro and in vivo dose-effects: a review. Pulm Pharmacol Ther 20: 9-22, 2007. 
28. Stav D and Raz M: Effect of N-acetylcysteine on air trapping in COPD: a randomized placebo-controlled study. Chest 136 : 381-386, 2009

29. Pela R, Calcagni AM, Subiaco S, et al: $\mathrm{N}$-acetylcysteine reduces the exacerbation rate in patients with moderate to severe COPD Respiration 66: 495-500, 1999.

30. Gerrits CM, Herings RM, Leufkens HG and Lammers JW: $\mathrm{N}$-acetylcysteine reduces the risk of re-hospitalisation among patients with chronic obstructive pulmonary disease. Eur Respir J 21: 795-798, 2003.

31. Millea PJ: N-acetylcysteine: multiple clinical applications. Am Fam Physician 80: 265-269, 2009

32. Hooper C and Calvert J: The role for S-carboxymethylcysteine (carbocisteine) in the management of chronic obstructive pulmonary disease. Int J Chron Obstruct Pulmon Dis 3: 659-669, 2008.

33. Zheng JP, Kang J, Huang SG, et al: Effect of carbocisteine on acute exacerbation of chronic obstructive pulmonary disease (PEACE Study): a randomised placebo-controlled study. Lancet 371: 2013-2018, 2008.

34. Yasuda H, Yamaya M, Sasaki T, et al: Carbocisteine reduces frequency of common colds and exacerbations in patients with chronic obstructive pulmonary disease. J Am Geriatr Soc 54: 378-380, 2006

35. Tatsumi K and Fukuchi Y; PEACE Study Group: Carbocisteine improves quality of life in patients with chronic obstructive pulmonary disease. J Am Geriatr Soc 55: 1884-1886, 2007.

36. Hauber HP and Zabel P: Emerging mucus regulating drugs in inflammatory and allergic lung disease. Inflamm Allergy Drug Targets 7: 30-34, 2008.

37. Li G, Cun X, Li L and Yongqing X: Advances in studies on Platycodon grandiflorum. China Journal of Chinese Materia Medica 32: 183-185, 2007 (In Chinese).

38. Bin Y, Yongqing X, Rixin L, Ruojing W, Wen L, Cun Z, Ying C, Qianpeng W, Lan W and Yongyan W: Studies on expectorant compounds in volatile oil from root and rhizome of Aster tataricus. China Journal of Chinese Materia Medica 33: 281-283, 2008 (In Chinese).

39. Haiqing W, Hongwei K, Kang J, Jingli S, Yan L, Jun T and Shiyou Y: Antitussive, expectorant and antibacterial actions of Zhebei Zhike granules. Chinese Journal of Experimental Traditional Medical Formulae 17: 207-210, 2011 (In Chinese).

40. McKay A, Leung BP, McInnes IB, et al: A novel anti-inflammatory role of simvastatin in a murine model of allergic asthma. J Immunol 172: 2903-2908, 2004

41. Weitz-Schmidt G: Statins as anti-inflammatory agents. Trends Pharmacol Sci 23: 482-486, 2002.

42. Wang HX, Sun B, Li JQ et al: Effect of simvastatin on airway mucus hypersecretion in rats and its molecular mechanism. Di 3 Jun Yi Da Xue Xue Bao 30: 213-215, 2008 (In Chinese).

43. Søyseth V, Brekke PH, Smith P and Omland T: Statin use is associated with reduced mortality in COPD. Eur Respir J 29 279-283, 2007.

44. Mancini GB, Etminan M, Zhang B, et al: Reduction of morbidity and mortality by statins, angiotensin-converting enzyme inhibitors, and angiotensin receptor blockers in patients with chronic obstructive pulmonary disease. J Am Coll Cardiol 47: 2554-2560, 2006

45. Kitazaki T, Fukuda M, Soda $\mathrm{H}$ and Kohno S: Novel effects of gefitinib on mucin production in bronchioloalveolar carcinoma; two case reports. Lung Cancer 49: 125-128, 2005.

46. Kitazaki T, Soda H, Doi S, et al: Gefitinib inhibits MUC5AC synthesis in mucin-secreting non-small cell lung cancer cells. Lung Cancer 50: 19-24, 2005.

47. Guan AY, Xu ZB, Wen FQ, Wang BD and Feng Y: Effect of gefinitib on airway mucus hypertension induced by acrolein in rats. Sichuan Da Xue Xue Bao Yi Xue Ban 39: 231-234, 2008 (In Chinese)

48. Vignola AM: PDE4 inhibitors in COPD - a more selective approach to treatment. Respir Med 98: 495-503, 2004

49. Kroegel $\mathrm{C}$ and Foerster M: Phosphodiesterase-4 inhibitors as a novel approach for the treatment of respiratory disease: cilomilast. Expert Opin Investig Drugs 16: 109-124, 2007.

50. Michalski JM, Golden G, Ikari J and Rennard SI: PDE4: a novel target in the treatment of chronic obstructive pulmonary disease. Clin Pharmacol Ther 91: 134-142, 2012.
51. Moretti M, Bottrighi P, Dallari R, et al; EQUALIFE Study Group: The effect of long-term treatment with erdosteine on chronic obstructive pulmonary disease: the EQUALIFE Study. Drugs Exp Clin Res 30: 143-152, 2004.

52. Marchioni CF, Polu JM, Taytard A, et al: Evaluation of efficacy and safety of erdosteine in patients affected by chronic bronchitis during an infective exacerbation phase and receiving amoxycillin as basic treatment (ECOBES, European Chronic Obstructive Bronchitis Erdosteine Study). Int J Clin Pharmacol Ther 33: 612-618, 1995.

53. Rhee CK, Kang CM, You MB, Yoon HK, Kim YK, Kim KH, Moon HS, Park SH and Song JS: Effect of fudosteine on mucin production. Eur Respir J 32: 1195-202, 2008.

54. Xin S, Yi S, Zenghua X, Yong S and Gui Z: A randomized controlled trial of expectorant in patients with respiratory tract infection. Chinese Journal of New Drugs and Clinical Remedies 25: 369-372, 2006 (In Chinese).

55. Li N, Li Q, Zhou XD, et al: The effect of quercetin on human neutrophil elastase-induced mucin5AC expression in human airway epithelial cells. Int Immunopharmacol 14: 195-201, 2012.

56. Wu H, Li Q, Zhou X, et al: Theaflavins extracted from black tea inhibit airway mucous hypersecretion induced by cigarette smoke in rats. Inflammation 35: 271-279, 2012.

57. Kwon SH, Nam JI, Kim SH, et al: Kaempferol and quercetin, essential ingredients in Ginkgo biloba extract, inhibit interleukin-1beta-induced MUC5AC gene expression in human airway epithelial cells. Phytother Res 23: 1708-1712, 2009.

58. Khan S, Liu YC, Khawaja AM, et al: Effect of the long-acting tachykinin NK(1) receptor antagonist MEN 11467 on tracheal mucus secretion in allergic ferrets. Br J Pharmacol 132: 189-196, 2001.

59. Shao MX and Nadel JA: Neutrophil elastase induces MUC5AC mucin production in human airway epithelial cells via a cascade involving protein kinase $\mathrm{C}$, reactive oxygen species, and TNF-alpha-converting enzyme. J Immunol 15: 4009-4016, 2005.

60. Chughtai B and O'Riordan TG: Potential role of inhibitors of neutrophil elastase in treating diseases of the airway. J Aerosol Med 17: 289-298, 2004

61. Feng L, Zhu W, Huang C and Li Y: Direct interaction of ONO-5046 with human neutrophil elastase through ${ }^{1} \mathrm{H}$ NMR and molecular docking. Int J Biol Macromol 51: 196-200, 2012.

62. Tomiyama H, Takara I, Tokumine J and Sugahara K: Sivelestat sodium hydrate was effective for ARDS in a patient suffering from chronic rheumatoid arthritis with acute exacerbation after failing to respond to high dose steroid pulse therapy. Masui 53: 1042-1046, 2004 (In Japanese).

63. Hubeau C, Singer M, Lagranderie M, et al: Extended freeze-dried Mycobacterium bovis Bacillus Calmette-Guérin induces the release of interleukin-12 but not tumour necrosis factor- $\alpha$ by alveolar macrophages, both in vitro and in vivo. Clin Exp Allergy 33: 386-393, 2003.

64. Cheng G, Arima M, Honda K, et al: Anti-interleukin-9 antibody treatment inhibits airway inflammation and hyperreactivity in mouse asthma model. Am J Respir Crit Care Med 166: 409-416, 2002.

65. Tomkinson A, Tepper J, Morton M, Bowden A, Stevens L, Harris P, Lindell D, Fitch N, Gundel R, Getz EB: Inhaled vs subcutaneous effects of a dual IL-4/IL-13 antagonist in a monkey model of asthma. Allergy 65: 69-77, 2010.

66. Cook ML and Bochner BS: Update on biological therapeutics for asthma. World Allergy Organ J 3: 188-94, 2010.

67. Sabater JR, Mao YM, Shaffer C, et al: Aerosolization of $\mathrm{P} 2 \mathrm{Y}(2)$-receptor agonists enhances mucociliary clearance in sheep. J Appl Physiol (1985) 87: 2191-2196, 1999.

68. Yerxa BR, Sabater JR, Davis CW, et al: Pharmacology of INS37217 [P(1)-(uridine 5')-P(4)- (2'-deoxycytidine 5')tetraphosphate, tetrasodium salt], a next-generation P2Y(2) receptor agonist for the treatment of cystic fibrosis. J Pharmacol Exp Ther 302: 871-880, 2002.

69. Kellerman DJ: P2Y(2) receptor agonists: a new class of medication targeted at improved mucociliary clearance. Chest 121 (5 Suppl): 201S-205S, 2002.

70. Poole P, Black PN and Cates CJ: Mucolytic agents for chronic bronchitis or chronic obstructive pulmonary disease. Cochrane Database Syst Rev 8: CD001287, 2012. 